\title{
ENERGIA DE FRACTURA: UN CONCEPTO NOVEDOSO PARA DETERMINAR LA RESISTENCIA EN VIGAS CON REBAJE. Victor
}

Carvallo A. Ingeniero Civil, División Industrias. Instituto Forestal, Santiago, Chile.

\section{RESUMEN}

Se entregan antecedentes respecto a los estudios realizados en el Instituto de Tecnología Lund de Suecia, relacionados con el comportamiento de vigas de madera son rebaje en su extremo (Gustafsson, 1988).

Los estudios están inspirados en la diferencia de fundamentos que se encuentra al comparar las fórmulas de diseño convencionales y los resultados téricos obtenidos mediante fractura mecánica y elementos finitos. Además, se desarrolla una ecuación simple para determinar la resistencia en este tipo de vigas, basada en la teoría de tractura mecánica.

Finalmente, se entrega la metodología para determinar empíricamente el parámetro fundamental en el diseño de vigas rebajadas mediante el concepto propuesto: la energía de fractura.

Palabras Clave: Madera, Vigas, Energía fractura, Rebaje. 


\section{INTRODUCCION}

Un elemento estructural se caracteriza fundamentalmente por su capacidad para resistir la combinación de cargas o solicitaciones a las que es sometido. De esta forma, una estructura está compuesta por un número limitado de elementos que, en su conjunto, son capaces de dar a ella la estabilidad adecuada, ponderando por medio del análisis estructural, las tensiones de trabajo y las de diseño para cada pieza y especie en forma individual.

La tensión de diseño se obtiene del producto entre la tensión admisible y los factores de modificación respectivos, de acuerdo a las condiciones particulares de carga y servicio. A su vez, la tensión admisible es el resultado de multiplicar la tensión básica por la razón de resistencia asignada a cada grado específico, con el fin de considerar sus defectos. Por último, la tensión básica se obtiene a través de un análisis estadístico de los valores que resultan de someter a ensayo, bajo distintos esfuerzos, a probetas estandarizadas libres de defectos.

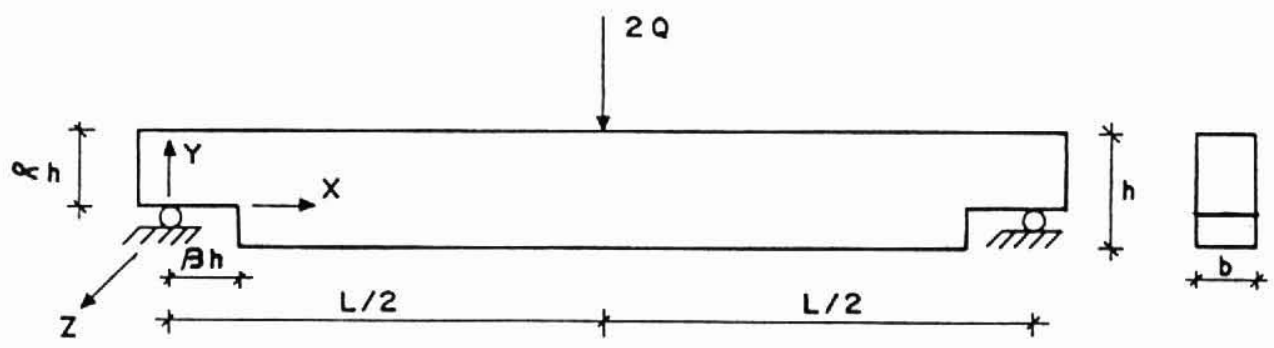

Figura No 1 VIGA CON REBAJE RECTANGULAR EN LOS EXTREMOS.

Como se puede apreciar, las tensiones de diseño son valores conocidos que los calculistas determinan con la ayuda de textos especializados, por lo que su estimación no representa gran problema.

Al incluir el otro parámetro en cuestión, la tensión de trabajo, el análisis se hace algo más complejo. La tensión de trabajo se define como la carga por 
unidad de superficie que expresa el esfuerzo interno real que tiene una sección de un elemento estructural en un momento dado. En consecuencia, se determina mediante las herramientas que entrega la mecánica de estructuras, tomando en cuenta para cada elemento en particular, la combinación de cargas a que es sometido, su sistema de apoyo y su geometría.

De esta forma se pueden individualizar los esfuerzos de trabajo en flexión, compresión, tracción, corte, etc., o sus combinaciones, y confrontarlos con sus similares de diseño, de manera tal que el cuociente entre la tensión de trabajo y la tensión de diseño sea menor que la unidad.

Un tipo de elemento considerado como estructural es la viga.Por lo general, el cálculo de las tensiones de trabajo a que una viga está sujeta no representa problemas desde el punto de vista teórico, sin embargo, usualmente y por razones constructivas, una viga puede ser rebajada en uno o ambos extremos, lo que ciertamente representa algún grado de incertidumbre debido a que las fórmulas convencionales para vigas con rebaje no tienen una teoría básica de sustentación. Estas fórmulas son numéricamente diferentes, pero análogas, y entregan la resistencia por simple reducción del esfuerzo de corte de diseño asociado a vigas sin rebaje o normales.

\section{FORMULAS CONVENCIONALES NORMADAS}

La fórmula más conocida para determinar la tensión de trabajo para el esfuerzo de corte máximo, se deriva de la expresión de momento estático o momento de inercia de primer orden. De ahi se deduce que:

$$
f_{c z}=\frac{3}{2} * \frac{Q}{b^{*} h} \quad \text { o } \quad \frac{Q}{b^{*} h}=\frac{2}{3} * f_{c z}
$$

Donde:

Q =Esfuerzo de corte máximo

b $\quad=$ Espesor de la viga 


$$
\begin{array}{ll}
\mathrm{h} & =\text { Altura de la viga } \\
\mathrm{f}_{\mathrm{cz}} & =\text { Tensión de trabajo máxima de corte longitudinal. }
\end{array}
$$

Ahora, si la ecuación anterior se expresa considerando el rebaje en la viga como una fracción de la altura, se tiene:

$$
\frac{Q}{b^{*} \alpha h}=\frac{2}{3} * f_{c z}^{*} f(\alpha)
$$

Donde $f(\alpha)$ es un factor de reducción que indica la influencia de la concentración de tensiones dependiente sólo de la profundidad del rebaje, frecuentemente $f(\alpha)=\alpha$

Se puede apreciar además que $Q$ es proporcional a $f_{c z}$.

En el EUROCODE 5 (1987) se encuentra otra ecuación, algo diferente a la (1), la que se usa para el diseño de vigas fabricadas con madera laminada encolada. En esa norma, se aplica un análisis Weibull para determinar el esfuerzo de corte en vigas sin rebaje, con un volumen mayor que $0,1 \mathrm{~m}^{3}$. Asi, el esfuerzo de corte se hace dependiente del volumen de la viga. Además, y al igual que en la ecuación (1), la resistencia de una viga con rebaje se toma como una fracción $\alpha$-dependiente de la resistencia de una viga sin rebaje.

$$
\frac{Q}{b^{*} \alpha h}=\frac{2}{3} * f_{c z} * f(\alpha) *\left(\frac{0,1 m^{3}}{V}\right)^{0,2} * \frac{1}{1-2 h / L}
$$

Donde :

$$
\begin{array}{ll}
\mathrm{V} & =\text { Volumen de la viga en } \mathrm{m}^{3} \\
\mathrm{~L} & =\text { Largo de la viga. }
\end{array}
$$

Conjuntamente con el volumen de la viga, el esfuerzo de corte también depende de $\mathrm{h} / \mathrm{L}$, lo que refleja la capacidad de poder incrementar la fuerza de corte si el tramo cizallado es muy corto.

En la norma australiana AS 1720-1975 se entrega una ecuación con fundamentos distintos a las dos anteriores. Dicha ecuación se basa en parte en la teoria de fractura mecánica: 


$$
\frac{Q}{b^{\star} \alpha h}= \begin{cases}\frac{2{ }^{*} f_{c z}}{\sqrt{h}\left(1+1,2^{*} \beta / \alpha\right)} & \text { para } \alpha \leq 0,9 \\ \frac{2 * f_{c z}}{3 \sqrt{(1-\alpha) * h} *(1+1,2 * \beta / \alpha)} & \text { para } \alpha>0,9\end{cases}
$$

Se deduce que, además de $\alpha, \mathrm{Q} /\left(\mathrm{b}^{*} \alpha \mathrm{h}\right)$ depende también de la longitud relativa del rebaje, $\beta, y$ de la altura absoluta de la viga, $h$ (en $\mathrm{mm}$ ).

La ecuación (3) es válida sólo para vigas rebajadas, pues la falla no puede ocurrir para $\alpha=1$ dado que $Q \rightarrow \infty$ cuando $\alpha \rightarrow 1$.

En la Figura $\mathrm{N}^{\circ} 2$ se comparan las tres ecuaciones anteriores mostrando $Q /\left(b^{*} \alpha h\right)$ versus $\alpha$ para $f_{c z}=10 \mathrm{MPa}\left(102 \mathrm{Kg} / \mathrm{cm}^{2}\right)$, en donde se puede apreciar la disparidad de soluciones para un problema similar. 


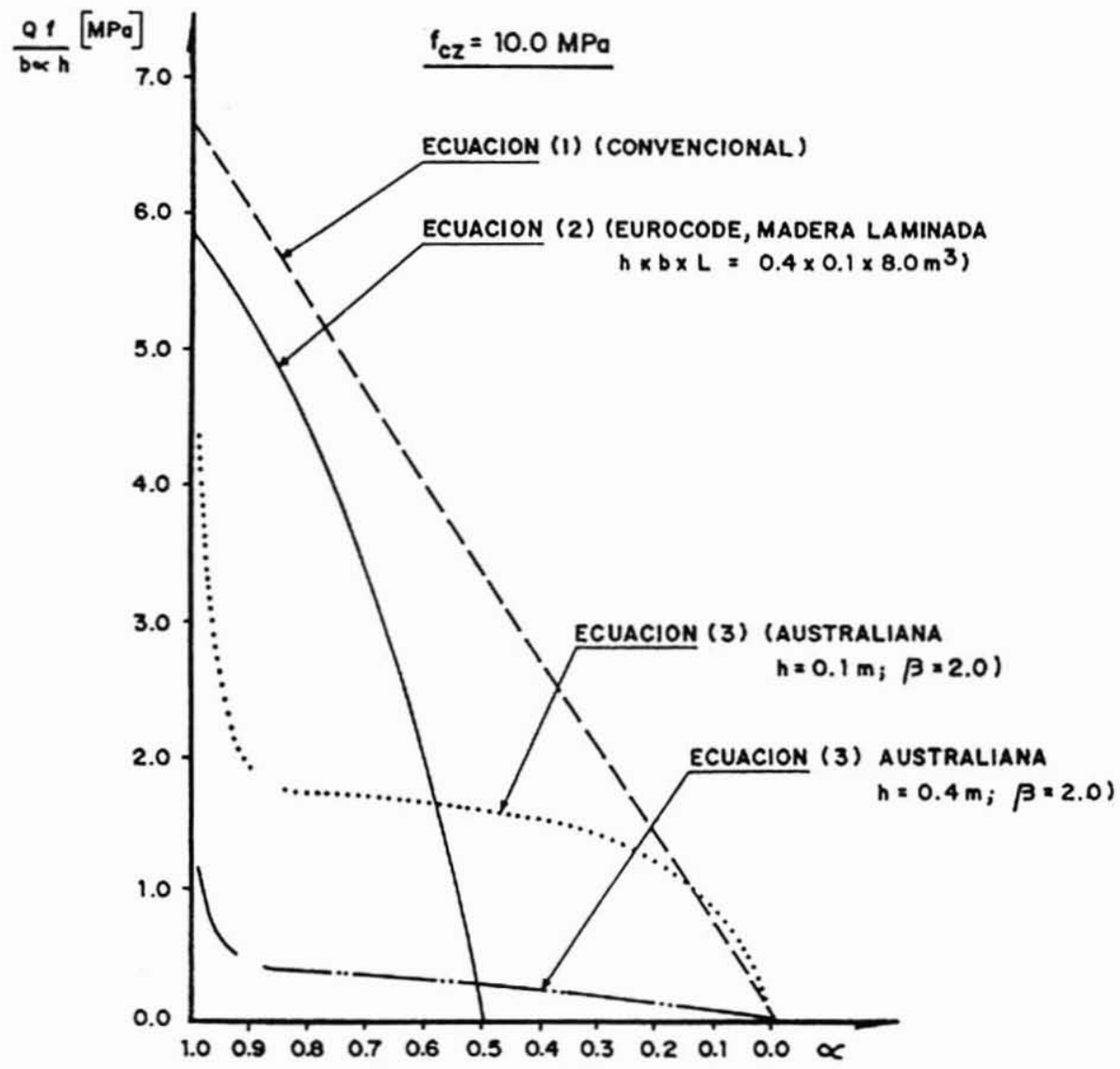

Figura Nº 2 .COMPARACION ENTRE FORMULAS CONVENCIONALES NORMADAS.

\section{ANALISIS TEORICO}

El análisis para ciertos esfuerzos máximos o criterios de resistencia son 
dificultosos, debido a la singularidad de la tensión en el extremo rebajado de una viga. Por tal singularidad, ninguna teoria Weibull parece dar alguna información significativa (Gustafsson y Enquist, 1988).

Por otra parte, el análisis mediante elementos finitos no es apropiado para el cálculo de este tipo de esfuerzo en un diseño ordinario, debido a requerimientos de simplicidad.

Sin embargo, es factible realizar un análisis teórico mediante fractura mecánica.

\section{Derivación de Expresiones Básicas}

Por consideración de balance de energía es posible obtener una ecuación para determinar la tensión en una viga rebajada ( mostrada en la Figura $N^{0} 1$ ). Asumiendo que el material es ortotrópico y linealmente elástico y que para una carga externa cero, todos los esfuerzos internos en la viga son también cero, entonces la energía potencial para el sistema carga externa y viga es:

$$
W_{p}=-\frac{1}{2} * Q * \delta
$$

Donde :

$\delta$ corresponde a la deflexión en el punto de carga.

Ahora, si para una fuerza constante, $Q$, se desarrolla una rajadura de largo $\Delta X$ desde el extremo rebajado, entonces el cambio de energía potencial, $\Delta W p$, debido al incremento en la deflexión, $\Delta \delta$, es:

$$
\Delta W_{p}=-\frac{1}{2}{ }^{*} Q{ }^{*} \Delta \delta=-\frac{1}{2} * Q^{2} \Delta(\delta / Q)
$$

Además se supone que la rajadura tiene un extremo preciso y que se desarrolla para una fuerza exacta de corte $Q=Q_{f}$ cuando la pérdida de energía potencial - $\Delta W p$ iguala la energia requerida para formar la rajadura. Esta energia, definida como energía de fractura, depende de las propiedades 
del material. Usando la nomenclatura $G_{c}$ para denotar energía de fractura/área de la rajadura, se obtiene:

$$
\frac{1}{2} * Q_{f}{ }^{2} \Delta(\alpha / Q)=G_{c} * b * \Delta X
$$

La parte de la viga que queda bajo la rajadura se considera inactiva, por lo cual no contribuye a la rigidez. De esta forma, el desarrollo de la rajadura, $\Delta X$, equivale a un incremento del largo del rebaje, desde $\beta$ h hasta $\beta h+\Delta X$. En consecuencia, el aumento de la rajadura se puede interpretar como un cambio $\Delta \beta$ de $\beta$. Esto es:

$$
\Delta \mathrm{X}=\mathrm{h}^{\star} \Delta \beta
$$

Usando esta última igualdad para $\Delta \mathrm{X} \rightarrow 0$, de la ecuación (6) se obtiene:

$$
\frac{Q_{1}}{b^{*} \alpha h}=\sqrt{\frac{2 G_{c}}{b^{*} \alpha^{2 * h}} / \frac{\partial(\delta / Q)}{\partial \beta}}
$$

Se debe calcular ahora la deflexión, para obtener $Q_{1}$ de acuerdo a la variación de $\beta$. En general, la deflexión de la viga se puede separar en cuatro términos:

$$
\delta=\delta_{1}+\delta_{Q}+\delta_{r}+\delta_{b}
$$

$\delta_{1}$ corresponde a la deformación local del material en los puntos de carga y apoyo. Se supondrá constante durante la variación de $\beta$ y, por ende, no se necesita calcular.

$\delta_{Q}$ representa la deformación por corte. Para una viga de sección rectangular se obtiene la siguiente variación lineal $\delta_{Q}$ en función de $\beta$ :

$$
\delta_{Q}=\frac{1,2^{*} Q}{G}\left[\frac{\beta^{*} h}{b^{*} \alpha^{*} h}+\frac{L / 2-\beta^{*} h}{b^{*} h}\right]
$$

Donde : 
G es el módulo de corte del material.

$\delta_{r} \quad$ corresponde al efecto elástico que el empotramiento del voladizo $\beta$ h, ejerce sobre el resto de la viga. Es razonable suponer que esta deformación es proporcional al momento flector, $Q \beta$ h, y al largo, $\beta$, del voladizo, con lo que se obtiene la variación de $\delta_{\mathrm{r}}$ en función de $\beta^{2}$ :

$$
\delta_{r}=c * Q * \beta^{2} * h^{2}
$$

En apariencia, una elección cómoda seria, $c=0$, sin embargo ello subestimaria la deflexión de la viga. Otra posibilidad mucho más acertada es:

$$
c=\frac{12}{b^{*}\left(\alpha^{*} h\right)^{2}} \sqrt{\frac{(1-\alpha)\left(1-\alpha^{3}\right)}{10^{*} G^{*} E_{x}}}
$$

Donde :

$$
E_{x} \text { es el módulo de elasticidad paralelo a la fibra. }
$$

Por último, $\delta_{b}$ representa la deformación normal de la viga, debido a la flexión. La teoria convencional entrega la variación de $\delta_{b}$ en función de $\beta^{3}$.

$$
\delta_{b}=\frac{Q}{E_{x}^{*} b^{*} h^{3} / 12}\left[\frac{L^{3}}{24}+\frac{(\beta h)^{3}}{3}\left(\frac{1}{\alpha^{3}}-1\right)\right]
$$

Luego, la deformación total de la viga (ecuación 9), se puede escribir de manera simple como:

$$
\alpha=A(B+\beta h)^{3}+K
$$

Donde :

$K$ es constante respecto a la variación de $\beta$. 


$$
\begin{aligned}
& A=\frac{Q}{3 * E_{x}^{*} b * h^{3} / 12}\left(\frac{1}{\alpha^{3}}-1\right) \\
& B=\sqrt[n]{\frac{E_{X}}{10 G} * \frac{(1 / \alpha-1)}{\left(1 / \alpha^{3}-1\right)}}
\end{aligned}
$$

Usando las ecuaciones (14), (15) y (16) en la ecuación (8) se obtiene una expresión explicita para determinar la resistencia en vigas rebajadas:

$$
\frac{Q_{f}}{b^{*} \alpha h}=\frac{\sqrt{G_{c} / h}}{\sqrt{0,6 \alpha(1-\alpha) / G}+\beta \sqrt{6\left(1 / \alpha-\alpha^{2}\right) / E_{x}}}
$$

o alternativamente, en términos del esfuerzo de corte convencional, se puede deducir que:

$$
f_{c z} \leq \frac{1,5 \sqrt{G_{c} / h}}{\sqrt{0,6 \alpha(1-\alpha) / G}+\beta \sqrt{6\left(1 / \alpha-\alpha^{2}\right) / E_{x}}}
$$

\section{DETERMINACION DE LA ENERGIA DE FRACTURA}

Como se ha visto, la expresión para determinar la resistencia en vigas rebajadas mediante el método propuesto, depende fundamentalmente del parámetro definido como energia de fractura, $\mathrm{G}_{\mathrm{c}}$. El valor que se obtenga para dicho parámetro está directamente relacionado con el método de ensayo, de ahi la importancia de estandarizar el procedimiento con el objeto de poder comparar los resultados que se obtengan en distintos laboratorios. 
Descripción del procedimiento de ensayo

El ensayo consta de tres piezas de madera, encoladas como indica la Figura $N^{\circ} 3$, de manera que el conjunto forme una pieza de sección transversal a $x$ b y largo 7 a.

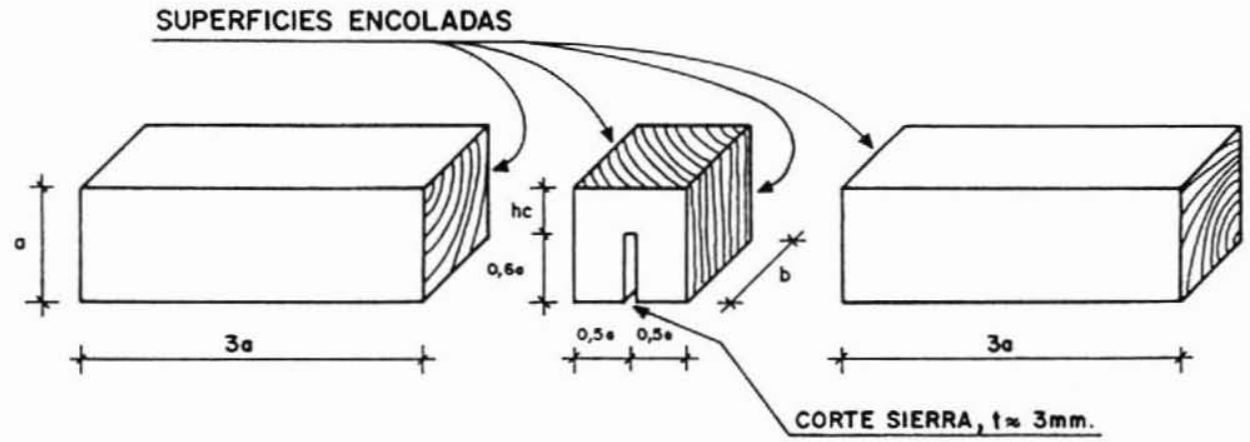

Figura No 3. PIEZAS QUE COMPONEN EL ENSAYO.

La probeta en la que se medirá la energia de fractura forma un prisma de volumen $a \times a \times b$, en la que se practica un corte con sierra, paralelo a dos de las caras paralelas a la fibra. Tal corte debe tener un espesor, $t$, de aproximadamente $3 \mathrm{~mm}$ y una profundidad de $0,6 \mathrm{a}$, de forma que el área ensayada sea $h_{c} \times b$, con $h_{c}=0,4 a$. 


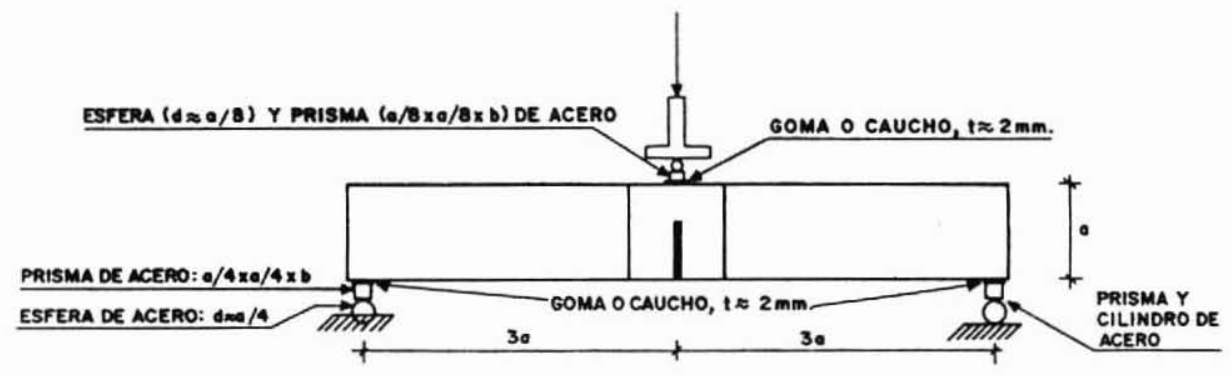

Figura $N^{\circ}$ 4. SISTEMA DE APOYO Y CARGA DE LA VIGA.

A la viga simplemente apoyada, se le aplica carga en el punto medio de la luz (ver Figura $\mathrm{N}^{\circ} 4$ ), a una velocidad constante del cabezal de la máquina de ensayo, de tal suerte que el colapso se obtenga en aproximadamente $(a / 80)$ $(3 \pm 1)$ min (a en $\mathrm{mm}$ ). Se debe registrar continuamente la carga y la deflexión con el objeto de determinar con exactitud el diagrama carga-deformación respectivo.

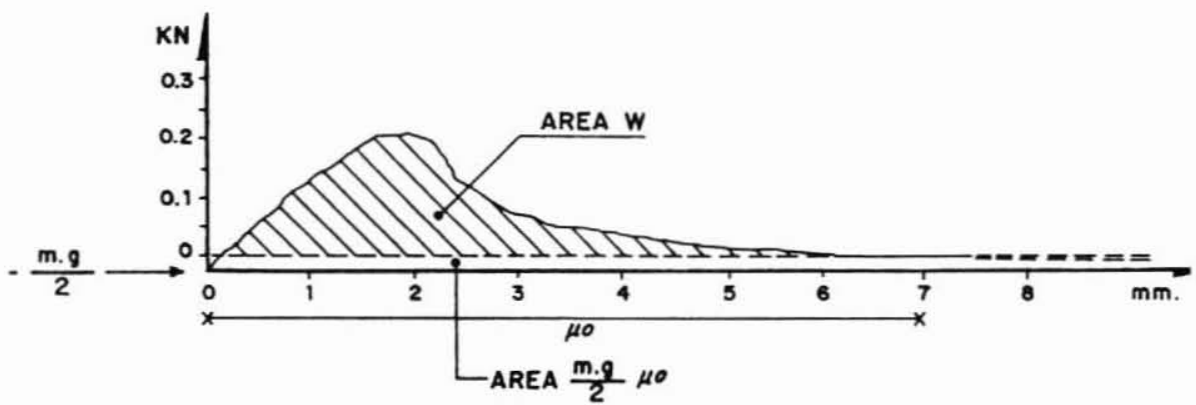

Figura No 5. DIAGRAMA CARGA - DEFORMACIÓN. 
Del diagrama carga-deformación, el trabajo externo se calcula como $\mathrm{W}+$ $m g \mu_{0}$, donde $W$ es el trabajo generado por la carga central, que se obtiene como el área bajo la curva mostrada en la Figura $N^{\circ} 5, m$ es la masa de la viga, g es la aceleración de gravedad y $\mu_{0}$ es la deformación hasta el punto de falla. El trabajo realizado por la masa de la viga, antes de $\mu_{\mathrm{o}}$ es $m g \mu_{\mathrm{o}} / 2 \mathrm{y}$, se asume que después de ese punto, la energía es de la misma magnitud.

Finalmente y de acuerdo a lo anterior, la energía de fractura se calcula como:

$$
G_{c}=\frac{W+m g \mu_{0}}{h_{c}^{*} b}
$$

\section{CONCLUSIONES}

El concepto de energía de fractura resuelve el problema relativo a la determinación de la resistencia en vigas rebajadas en sus extremos de manera simple. Sus fundamentos son sólidos, pues está incorporada dentro de una teoria consistente (fractura mecánica), que considera la situación real de carga, geometría y apoyo del elemento estructural, concentrando el análisis en el verdadero punto critico que puede llevar al colapso, esto es, la potencial rajadura longitudinal que se inicie en el vértice del rebaje.

Ello entrega a los calculistas, una importante herramienta que hace más confiable el análisis estructural y, si es el caso, la posibilidad de determinar con exactitud el esfuerzo predominante.

Por otra parte, esta proposición fue discutida en el Congreso CIB W18 (International Council for Building Research Studies and Documentation. Working Commission W18 A - Timber Structures), en Septiembre de 1988, acordándose en aquella oportunidad, que ella sería incorporada en la futura versión de la norma de Diseño de Estructuras en Madera del ClB.

Lo anterior, lleva a pensar que en el corto plazo, esta metodologia de diseño para vigas rebajadas forme parte de, al menos, la Norma Europea, pasando a constituir parte del conjunto de parámetros que se exigen, como información 
básica, para la exportación de madera con cualidades estructurales. De ahí la importancia que en Chile se investigue la energía de fractura de las especies forestales crecidas en el país.

\section{BIBLIOGRAFIA}

EUROCODE 5, 1987. Common Unified Rules for Timber Structures. Commission of the European Communities, Report EUR 9887.

Gustafsson, Per Johan, 1988. A Study of Strength of Notched Beams. CIB-W18 Paper 21-10-1, Parksville, Canada. 1988 a.

Larsen, H. J. and Gustafsson P. J, 1989. Design of Endnotched Beams. CIB-W18 A Paper 22-10-1, Berlin, German Republic.

Smith, Seely, 1970. Resistencia de Materiales, Segunda Edición, Editorial HispanoAmericana, México.

Walpole, R. E. and Myers, R. H., 1989. Probability and Statistics for Engineers and Scientists. Fourth Edition. Mac Millan Publishing Company. New York, U.S.A. 